\title{
A linear least-squares version of the algorithm of mode isolation for identifying modal properties. Part I: Conceptual development
}

\author{
Jerry H. Ginsberg ${ }^{\text {a) }}$ and Matt Allen \\ G. W. Woodruff School of Mechanical Engineering, Georgia Institute of Technology, \\ Atlanta, Georgia 30332-0405
}

(Received 28 October 2003; revised 23 April 2004; accepted 5 May 2004)

\begin{abstract}
The Algorithm of Mode Isolation (AMI) is an iterative procedure for identifying the number of modes contributing to a frequency response function (FRF) concurrently with identifying the complex eigenvalues and eigenvectors of those modes. The latest modifications obtain these modal properties solely by using linear least squares fits of the FRF data to canonical forms. The algorithmic operations are explained in a detailed sequence of steps that are illustrated by some sample data. The computational efficiency of AMI relative to other modal identification algorithms that fit response data to multi-degree-of-freedom model equations is discussed. () 2004 Acoustical Society of America. [DOI: 10.1121/1.1765195]
\end{abstract}

PACS numbers: 43.40.Le, 43.40.Cw, 43.40.Yq [JGM]

Pages: $900-907$

\section{INTRODUCTION}

Algorithms for experimental modal analysis of linear dynamic systems can be categorized as to whether they use frequency or time domain data. Another categorization addresses the analytical representation of a response to which the measured data is fit. Single-degree-of-freedom (SDOF) techniques consider modes to act independently, whereas multiple-degree-of-freedom (MDOF) techniques allow for the modal contributions to overlap. A third descriptor pertains to the number of locations for excitation and response measurement. Single input-single output (SISO) uses a single pair, while multiple input-single output (MISO) uses multiple excitations and measures response at one location. If the system is time invariant, so that the principle of reciprocity applies, then single input-multiple output (SIMO) is equivalent to MISO. Multiple input-multiple output (MIMO) uses the data obtained from a multitude of excitations and response measurements. The texts by Maia et al. ${ }^{1}$ and Ewins ${ }^{2}$ provide a good background for these concepts.

Modal properties to be identified are the natural frequencies and modal damping ratios, which are system properties that are independent of the selection of points at which the data is measured. Mode vectors also require determination, but their values are dependent on the locations of the measurement points. Underlying this need is the requirement to identify the number of modes active in a frequency band, or equivalently, the system order associated with a time domain response. MDOF algorithms, which seem to be most commonly employed, make an a priori guess for the number of modes. One approach for verifying the correctness of that guess is to examine a metric that indicates how well a reconstruction of the response using the identified modal properties fits the measured response. However, because the frequency response functions (FRFs) corresponding to a different excitation feature different weightings of the modal

${ }^{a)}$ Electronic mail: jerry.ginsberg@me.gatech.edu parameters, it does not follow that parameters giving a good metric for one set of FRFs are suitable for other excitations. An alternative for verifying the system order displays in stabilization charts the natural frequencies identified from a range of guesses, then discards modes that are not consistently obtained. ${ }^{3}$ This practice often requires considerable expertise of the analyst, and increases the computational effort. Also, it is best to overestimate the number of modes, but doing so raises the computational effort; it can also lead to false estimations and split modes, as was shown by Doebling, Alvin, and Peterson. ${ }^{4}$

Even if the number of participatory modes were known, the presence of relatively large damping can give rise to identification difficulties for several reasons. Some algorithms implicitly assume that dissipation is viscous. Furthermore, if a system has a wide range of modal damping ratios, the more highly damped modes in any transient temporal responses are rapidly attenuated, thereby magnifying the contribution of noise to those modes. For frequency domain data, high damping lowers the resonant peak of a frequency response function (FRF). Both serve to make it more difficult to distinguish the response from ambient noise, especially for in situ applications such as health monitoring applications. A further complication of large damping arises when natural frequencies are close, which is a common situation for highfrequency modes in complex systems. In such situations, the modal bandwidth of adjacent resonant peaks might exceed the natural frequency difference, leading to a merger of the resonant peaks into one broader peak, which is known as mode coupling. This can make it difficult to distinguish the individual modes.

For any identification technique proper selection of the drive point(s) is a primary factor affecting the quality of the identification. If the drive point mobility in a mode is small, that mode will participate little in the measured response, making it difficult to extract the mode. This situation is exacerbated by the presence of substantial noise, where one 
would expect that it is necessary for the response to stand out from the ambient noise.

Previous papers have suggested that the Algorithm of Mode Isolation (AMI) has the potential to address these difficult issues. It recognizes that several modes might simultaneously contribute to a FRF at any frequency, but does so in an iterative manner, in the course of which one obtains the number of modes active in any frequency interval. The algorithm was initially described by Drexel and Ginsberg ${ }^{5}$ for a classical, undamped, modal analysis, although the basic concept was previously mentioned by Joh and Lee. ${ }^{6}$ It then was extended to modal analysis in the state space in order to account for arbitrary damping (Drexel and Ginsberg, ${ }^{7}$ Drexel, Ginsberg, and $\mathrm{Zaki}^{8}$ ). In its original version AMI was a SISO algorithm, in that each FRF was processed individually. In order to improve the ability to identify modes that are poorly excited $\mathrm{Zaki}^{10}$ modified AMI by deferring identification of the normal mode coefficients. In the modified approach the original AMI algorithm is solely used to extract the eigenvalues, after which a global SIMO/MISO approach using a linear least-squares procedure identifies normalized mode vectors.

The heart of the AMI algorithm is to fit iteratively a FRF to the canonical form of a single mode. The aforementioned investigations used a nonlinear least squares routine to fit the data, which was the best available approach. It required good starting values, and entailed significant computational effort. Even then, convergence could be slow in some situations, and the results less accurate than desired. Ginsberg et al. ${ }^{11}$ presented a general SDOF fitting procedure, in which linear least squares is used to match a resonant peak in an FRF to the FRF of a complex mode. That technique is not iterative, and it yields an exact match for the analytical FRF of a SDOF system. Implementation of this fitting procedure in AMI substantially increases the reliability and computational efficiency of the individual iterative steps.

The complete elimination of nonlinear least squares routines within AMI, coupled with separate identification of eigenvalues and complex mode vectors, are significant alterations. In the present paper we provide a detailed description of the latest version. In Part $\mathrm{II}^{1}$ we assess its performance in comparison to analytical results, as well as relative to a popular modal identification algorithm.

\section{THE STANDARD MODE ISOLATION ALGORITHM}

The data to be input to AMI are the FRFs $H_{j P}\left(\omega_{m}\right)$, which are defined as the complex amplitudes of a set of generalized displacements $q_{j}$ when a specific generalized force $Q_{P}$ has a unit amplitude over a discrete set of frequencies $\omega_{m}$, and all other generalized forces are zero,

$Q_{j}=\operatorname{Re}\left[\delta_{j P} \exp \left(i \omega_{m} t\right)\right] \Rightarrow q_{j}=\operatorname{Re}\left[H_{j P}\left(\omega_{m}\right) \exp \left(i \omega_{m} t\right)\right]$.

By reciprocity, $H_{j P}\left(\omega_{m}\right)=H_{P j}\left(\omega_{m}\right)$. Hence, regardless of whether measurements are taken according to a SIMO or MISO protocol, the input data for AMI can be considered to be a rectangular array of FRF values, in which column $j$ holds the FRF values over a discrete set of frequencies $\omega_{m}$ associated with displacement $j$ and specified drive point $P$.
A FRF may be expressed as a finite sum of contributions of complex modes associated with a state-space description. If $N$ is the number of degrees of freedom, then there are $2 N$ eigensolutions, consisting of an eigenvalue $\lambda_{k}$ and eigenvector $\left\{\Phi_{k}\right\}$ that satisfy the symmetric eigenvalue problem,

$$
\left[\left[\begin{array}{cc}
{[0]} & {[K]} \\
{[K]} & {[C]}
\end{array}\right]-\lambda_{k}\left[\begin{array}{cc}
{[K]} & {[0]} \\
{[0]} & -[M]
\end{array}\right]\left\{\Phi_{k}\right\}=\{0\} .\right.
$$

The eigenvectors $\left\{\Phi_{k}\right\}$ are normalized according to

$$
\left\{\Phi_{k}\right\}^{T}\left[\begin{array}{cc}
{[K]} & {[0]} \\
{[0]} & -[M]
\end{array}\right]\left\{\Phi_{k}\right\}=1 \text {. }
$$

It is assumed that all eigenvalues occur as complex conjugate pairs. This corresponds to modes that are underdamped, meaning that their free response is oscillatory within an exponentially decaying window. By analogy with a SDOF system, an undamped natural frequency $\Omega_{k}$ and modal damping ratio $\zeta_{k}$ can be extracted from an eigenvalue according to

$$
\Omega_{k}=\left|\lambda_{k}\right|, \quad \zeta_{k}=-\operatorname{Re}\left(\lambda_{k}\right) /\left|\lambda_{k}\right| .
$$

If all eigenvalues are complex conjugates, the system may be considered to possess $N$ underdamped modes, whose contribution to any response may be represented by evaluating the role of the $N$ eigensolutions whose eigenvalues have positive imaginary parts, then using the conjugate property to account for the other $N$ eigensolutions. It is convenient in that case to sequence the eigenvalues such that those having positive imaginary parts come first followed by the conjugate values in the matching sequence. When this is done, each eigenvector may be expressed in terms of one of a set of $N$ column vectors $\left\{U_{k}\right\}$ whose $N$ elements represent the (complex) modal proportions of the generalized coordinates, according to

$$
\lambda_{k+N}=\lambda_{k}^{*} \Rightarrow\left\{\Phi_{k}\right\}=\left\{\begin{array}{c}
\left\{U_{k}\right\} \\
\lambda_{k}\left\{U_{k}\right\}
\end{array}\right\}, \quad\left\{\Phi_{k+N}\right\}=\left\{\Phi_{k}\right\}^{*}
$$

As a result of the conjugate properties of the complex modes, a FRF value may be expressed ${ }^{12}$ as a superposition of modal contributions according to

$$
H_{j P}(\omega)=\sum_{k=1}^{N}\left[\frac{A_{j P, k}}{i \omega-\lambda_{k}}+\frac{A_{j P, k}^{*}}{i \omega-\lambda_{k}^{*}}\right],
$$

where ( $)^{*}$ denotes a complex conjugate, and $A_{j P, k}$ are residue factors that depend solely on the eigensolution for mode $k$,

$A_{j P, k}=\lambda_{k} U_{j k} U_{P k}$

The foregoing is the analytical perspective, whereas in experimental modal analysis one does not know the number of degrees of freedom. Henceforth, $N_{0}$ will denote the number of measurement points for which FRFs are available, and $N$ will denote the number of modes whose natural frequency falls in the frequency interval covered by the FRF data. The determination of $N$ is a key aspect of any modal identification.

AMI begins by sequentially processing each of the $N_{0}$ FRF datasets. Two processes are applied to this data, both of 
which use current estimates of the eigenvalues and residue factors to subtract estimated modal contributions from the original data. The term "subtraction residual," denoted as $Y_{j, k}(\omega)$, refers to the data obtained by subtracting from $H_{j P}(\omega)$ estimated contributions for modes 1 to $k$, where the modes are numbered in the sequence in which they are detected. This data may be evaluated incrementally, such that

$$
\begin{aligned}
& Y_{j, 0}\left(\omega_{m}\right)=H_{j P}\left(\omega_{m}\right), \\
& Y_{j, k}\left(\omega_{m}\right)=Y_{j,(k-1)}\left(\omega_{m}\right)-\left[\frac{A_{j P, k}}{i \omega_{m}-\lambda_{k}}+\frac{A_{j P, k}^{*}}{i \omega_{m}-\lambda_{k}^{*}}\right] .
\end{aligned}
$$

In the Subtraction Phase, modes are identified one at a time, and their contribution to the FRF is subtracted until all significant modes have been removed. These operations may be summarized by a sequence of algorithmic steps.

(S.1) Pick a generalized displacement $j$ and initialize the subtraction residual $Y_{j, 0}\left(\omega_{m}\right)$ to be the FRF values for this coordinate at a discrete set of frequencies. Initialize the mode number as $k=0$.

(S.2) Identify the frequency range of the most dominant mode in the subtraction residual by searching for $\max \left(Y_{j, k}\left(\omega_{m}\right)\right)$. Use a SDOF fit of the data in this frequency range to estimate the most dominant mode's properties. (This step now uses the linear least-squares procedure presented by Ginsberg et al. ${ }^{11}$ and summarized in a later section.) Increment $k$ by one, and assign the identified modal parameters as $\lambda_{k}$ and $A_{j P, k}$.

(S.3) Form subtraction residual data $Y_{j, k}\left(\omega_{m}\right)$ according to Eq. (8).

(S.4) Decide whether $Y_{j, k}\left(\omega_{m}\right)$ contains the contribution of another mode. (The criteria for this decision are discussed below.) If so, return to Step (S.2). If $Y_{j, k}\left(\omega_{m}\right)$ consists solely of noise, then set the initial estimate for the number of modes to be $N=k$, and proceed to the Isolation Phase.

A troublesome aspect of the residual in Step (S.3) was recognized recently. The modal parameters identified in Step (S.2), being initial estimates, might differ significantly from the true values. Depending on their quality and the noise level, the residual $Y_{j, k}\left(\omega_{m}\right)$ formed from them might still contain an identifiable peak. This would cause the peak search in Step (S.2) to eventually return to this mode, for which an initial guess has already been obtained. The consequence would be multiple distinct initial estimates of a single mode. A simple way to avoid having the subtraction phase "chase its tail" is to zero $Y_{j, k}\left(\omega_{m}\right)$ over a range of $\omega_{m}$ surrounding the natural frequency, $\left|\lambda_{k}\right|$, that was identified.

The range for this zeroing must be selected carefully. If it is too big, it will obliterate the FRF in the vicinity of adjacent modes, whereas zeroing over an interval that is too small might leave significant spikes at the shoulders of the resonance peak. The range that was found to be effective is suggested by trials of the linear least squares identification algorithm described later. It was found for two-degree-offreedom systems that the two complex modes could be distinguished if the difference of their adjacent undamped natu- ral frequencies significantly exceeds $40 \%$ of the average bandwidth. Modes that are not distinguished because of their proximity are essentially treated as one mode in any SIMO/ MISO identification scheme. In view of this limitation, zeroing out the residual FRF over $80 \%$ of its bandwidth would only remove data containing modes that could not be distinguished from the one just found. This leads to the insertion of another operation after Step (S.3).

(S.3a) Set the subtraction residual data $Y_{j, k}\left(\omega_{m}\right)=0$ for $0.8 \operatorname{Re}\left(\lambda_{k}\right)+\left|\lambda_{k}\right|<\omega_{m}<-0.8 \operatorname{Re}\left(\lambda_{k}\right)+\left|\lambda_{k}\right|$.

The Isolation Phase has the primary purpose of accounting for the fact that more than one mode may contribute to a FRF. It uses current estimates for the modal parameters to isolate the contribution of a specific mode. This requires forming an "isolation residual," denoted $X_{j, k}\left(\omega_{m}\right)$, in which the current estimated contributions of all modes other than number $k$ are subtracted from $H_{j P}\left(\omega_{m}\right)$ according to

$$
X_{j, k}\left(\omega_{m}\right)=H_{j P}\left(\omega_{m}\right)-\sum_{\substack{n=1 \\ n \neq k}}^{N}\left[\frac{A_{j P, n}}{i \omega_{m}-\lambda_{n}}+\frac{A_{j P, n}^{*}}{i \omega_{m}-\lambda_{n}^{*}}\right] .
$$

The estimated modal properties required to begin the isolation process are those at the end of the Subtraction Phase, and the sequence in which the modes are isolated matches that in which the modes were identified in the Subtraction Phase. The steps required to carry out these operations are as follows.

(I.1) Initialize the mode number $k=0$.

(I.2) Increment $k$ by one, and form isolation residual data $X_{j, k}\left(\omega_{m}\right)$ according to Eq. (9). For this, use the latest values for $\lambda_{k}$ and $A_{j P, k}$.

(I.3) Apply the linear least-squares SDOF fit to $X_{j, k}\left(\omega_{m}\right)$ in order to obtain refined estimates of $\lambda_{k}$ and $A_{j P, k}$. Retain the previous values for convergence tests.

(I.4) If $k<N$, return to Step (I.2). If $k=N$, compare the new values and old values of all $\lambda_{k}$ and $A_{j P, k}$. If these values have not converged, return to Step (I.1). (Iterating until the real and imaginary parts of $\lambda_{k}$ and $A_{j P, k}$ change by less than $0.01 \%$ yields good results. For modes whose natural frequency differs from those of adjacent modes by more than the modal bandwidth, no more than five iterations are typically required.)

(I.5) Use the converged set of values of all $\lambda_{k}$ and $A_{j P, k}$ to form subtraction residual $Y_{j, N}\left(\omega_{m}\right)$, which accounts for all modes identified thus far.

(I.6) Decide whether $Y_{j, N}\left(\omega_{m}\right)$ contains the contribution of a (previously unidentified) mode. If not, cease processing of the FRF for the $j$ th displacement, and return to Step (S.1) to process another set of FRF data.

(I.7) This step is reached if there is evidence of an additional mode in $Y_{j, N}\left(\omega_{m}\right)$. Apply the linear least squares SDOF fit to this data in order to obtain initial estimates of $\lambda_{N+1}$ and $A_{j P,(N+1)}$.

(I.8) Increment $N$ by one, then set $k=N-1$ and return to Step (I.1). 

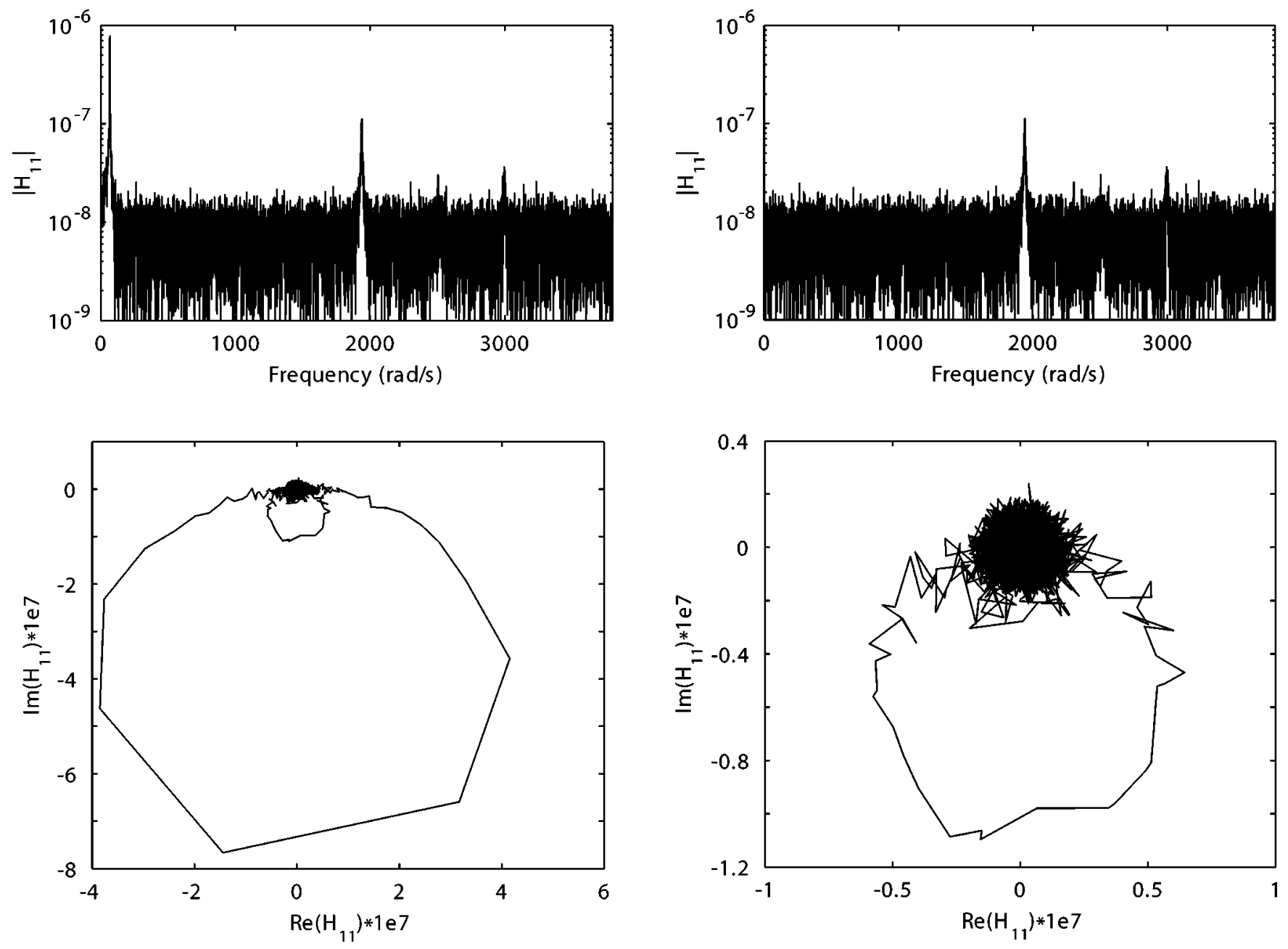

FIG. 1. Typical subtraction residual after removal of the first five modes.

All of the preceding operations are straightforward, except for Steps (S.4) and (I.6), which require a decision as to whether a residual FRF contains the contribution of a mode. If all of the modal contributions have been subtracted, the residual FRF should appear to be incoherent. The present procedure requires that the user visually search plots of the data to determine if there is some degree of coherence, e.g., several points form a noticeable peak in a Bode magnitude plot and a regular arc in a Nyquist plot. The identification of a quantitative measure on which to base this decision is a current area of investigation.

A useful perspective for the isolation stage is obtained by recognizing that because the isolation residual is formed by subtracting the current estimates for the contribution of known modes other than the one in focus, what remains consists of several parts: the actual contribution of the current mode, noise, measurement error, and errors associated with using parameters for the other modes that are not exact. Any of the latter that stand out above the noise will influence successive isolation steps until they are driven below the noise level. A corollary is that the noise floor defines the weakest modes that can be identified.

As an illustration of the Subtraction Phase, the latter stages of the processing of one of the FRFs described in Part II shall be followed here. The upper part of Fig. 1 shows the

FIG. 2. Typical subtraction residual after removal of the first six modes.

Bode magnitude plot of a residual FRF $\left|H_{11}(\omega)\right|$ after five modes have been identified and their contributions subtracted, that is, Step (S.4) with $k=5$. The lower part of Fig. 1 is a Nyquist plot of the same data. The irregularity of the data is a consequence of the white noise that was added to the data. Eleven modes have their natural frequency in the band covered by this figure, and five have been subtracted. The effect of the noise is to mask some of the six remaining modes. The Bode plot displays three peaks, but the Nyquist plot shows only two loops. (Each mode can be expected to produce a loop in a Nyquist plot. ${ }^{2}$ ) The presence of peaks in the Bode plot and coherent loops in the Nyquist plot indicates that the Subtraction Phase should continue, so the procedure passes to Step (S.2) with $k=6$. The peak below 100 $\mathrm{rad} / \mathrm{s}$ is the highest. The linear least squares routine is used to fit the data in the vicinity of this peak, thereby identifying the sixth eigenvalue and residue. The subtraction residual is computed in Step (S.3), and plotting of this data in accord with Step (S.4) leads to Fig. 2. The low-frequency peak has been eliminated, and there is only one loop in the Nyquist plot. Because there is still evidence of the presence of a mode in the FRF data, the procedure returns to Step (S.2) with $k=7$. The highest peak in the plot of $\left|H_{11}(\omega)\right|$ versus $\omega$ now lies in the vicinity of $2000 \mathrm{rad} / \mathrm{s}$, so the data in that region is used for the parameter identification, which yields 

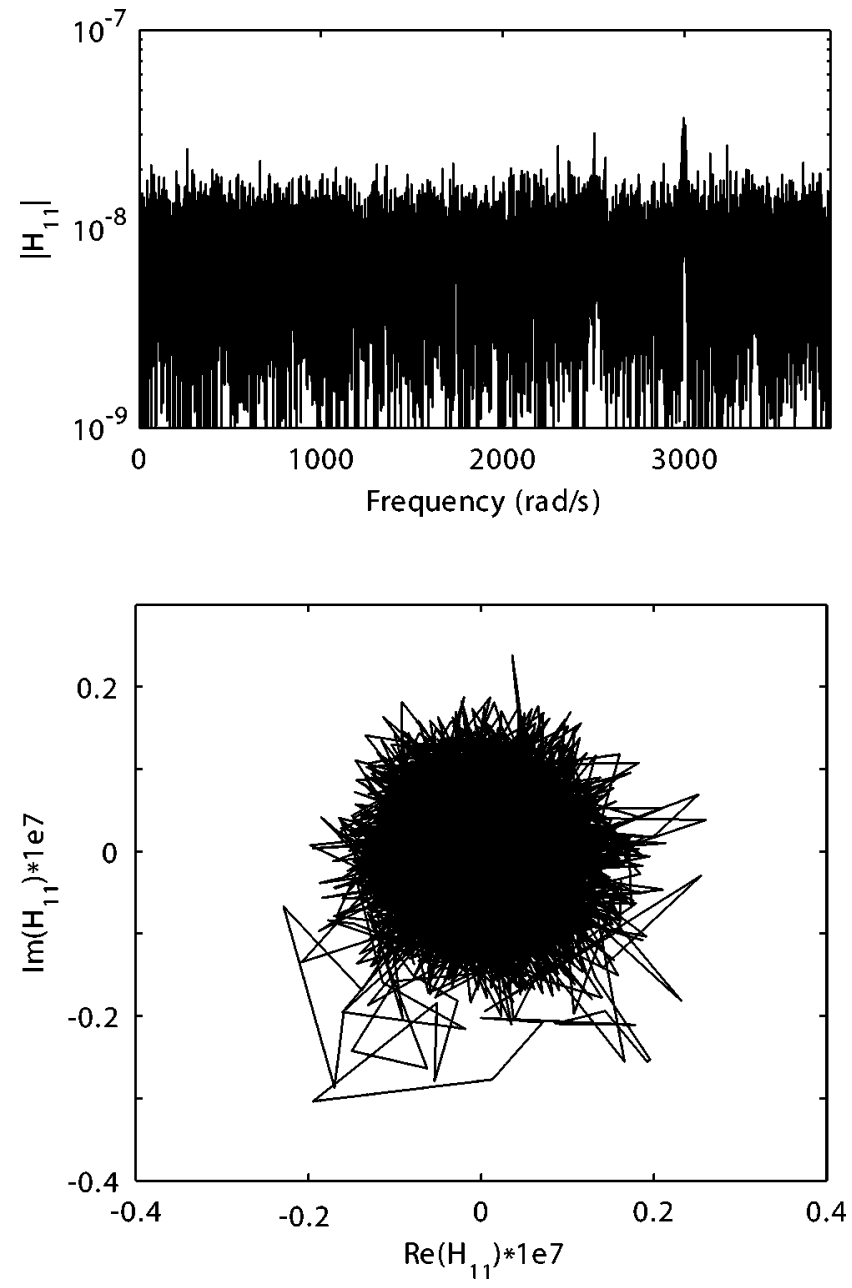

FIG. 3. Typical subtraction residual after removal of the first seven modes.

the seventh eigenvalue/residue estimate. Computing the subtraction residual FRF in Step (S.3) leads to the plots in Fig. 3, which are examined in Step (S.4). Although the Bode plot shows evidence of a small peak in the vicinity of $3000 \mathrm{rad} / \mathrm{s}$, the Nyquist plot shows no evidence of a regular arc. This indicates that the data is incoherent, so even if the peak actually corresponds to a mode, the identification of its properties would be unreliable. Consequently, the search is discontinued, and processing proceeds to the Isolation Phase with $N=7$.

Under ideal circumstances, processing the FRF for the $j$ th displacement coordinate as described in the preceding would give an estimate of $\lambda_{k}$ and $A_{j P, k}$ for $k=1, \ldots, N$. Subjecting each displacement dataset to the same processing then would lead to $N_{0}$ estimates of each of the $\lambda_{k}$ values, and a single estimate of each $A_{j P, k}$ coefficient for $j=1, \ldots, N_{0}$. In such ideal circumstances, each set of $\lambda_{k}$ for fixed $k$ would be averaged. However, as illustrated by Figs. 1-3, weakly excited modes or modes whose amplitude is small at the measurement location actually might not be identified in a specific FRF dataset. If such is the case, the missing values are merely omitted when average eigenvalues are computed.

There also might be noticeable discrepancies between corresponding eigenvalues obtained from different FRF sets. This leads to the question of whether the individual estimates

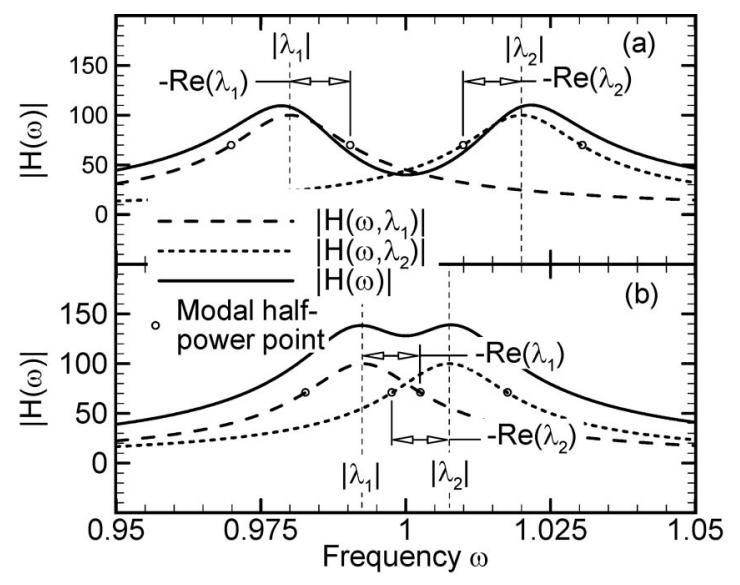

FIG. 4. Frequency response function formed by summing the FRFs of two modes having close natural frequencies. (a) $A_{1 P, 1}=A_{1 P, 2}=1, \lambda_{1}=-0.01$ $+0.98 i, \quad \lambda_{2}=-0.01+1.02 i$; (b) $A_{1 P, 1}=A_{1 P, 2}=1, \quad \lambda_{2}=-0.01+0.9975 i$, $\lambda_{2}=-0.01+1.0025 i$.

should be merged by averaging, or whether differences of estimates are sufficiently large to consider the eigenvalues to be distinct. The criterion that was implemented compares the difference of adjacent natural frequencies, $\left|\lambda_{k+1}\right|-\left|\lambda_{k}\right|$, to the modal bandwidths. For a single mode, the bandwidth is $-2 \operatorname{Re}\left(\lambda_{k}\right){ }^{12}$ Subtracting from the frequency difference the half-bandwidth for each mode yields the frequency interval between the adjacent half-power points belonging to two peaks. This is illustrated in Fig. 4, where a FRF $H(\omega)$ is synthesized from the contributions $H\left(\omega, \lambda_{1}\right)$ and $H\left(\omega, \lambda_{2}\right)$ of two neighboring modes having equal residues. In Fig. 4(a), $\left|\lambda_{2}\right|-\left|\lambda_{1}\right|$ is greater than the sum of the halfbandwidths, and the peaks of the FRF are quite distinct. In Fig. 4(b) $\left|\lambda_{2}\right|-\left|\lambda_{1}\right|$ is less than the sum of the halfbandwidths, which means that the bandwidths overlap. The peak FRF is a merger of the individual peaks, with a flattened region that shows a dimple. The sum of the halfbandwidths is the average bandwidth. Modal overlap corresponds to an average bandwidth that substantially exceeds the frequency difference. Experience has shown that the linear least squares identification algorithm described later usually will distinguish between eigenvalues in a single FRF if the difference of adjacent undamped natural frequencies significantly exceeds $40 \%$ of the average bandwidth. Based on this observed behavior, eigenvalues obtained from different FRFs are considered to be distinct if they fit a similar specification, specifically,

$$
\left|\lambda_{k+1}\right|-\left|\lambda_{k}\right|>0.4\left[-\operatorname{Re}\left(\lambda_{k}\right)-\operatorname{Re}\left(\lambda_{k+1}\right)\right] \text {. }
$$

Conversely, adjacent eigenvalues are averaged if they do not meet this specification. It should be noted that incorrectly merging modes having distinct eigenvalues results in their being treated as two (or more) modes that share the same eigenvalue. The full identification of such modes requires a MIMO algorithm; see Maia et al. ${ }^{1}$

\section{SINGLE MODE PARAMETER IDENTIFICATION}

Both the Subtraction and Isolation Phases require that one identify the properties of a single mode that best fit the FRF data in the vicinity of a resonance peak. Ginsberg 
et al. ${ }^{11}$ derived a linear least squares procedure for this purpose. The first step is to combine the two terms associated with a single mode in Eq. (6). This gives

$$
H_{j P}(\omega)=2 \frac{\left[i \omega-\operatorname{Re}\left(\lambda_{k}\right)\right] \operatorname{Re}\left(A_{j P, k}\right)-\operatorname{Im}\left(\lambda_{k}\right) \operatorname{Im}\left(A_{j P, k}\right)}{\left|\lambda_{k}\right|^{2}-\omega^{2}-2 i \omega \operatorname{Re}\left(\lambda_{k}\right)} .
$$

Clearing the denominator in Eq. (11) and breaking the result into real and imaginary parts then leads to

$$
\begin{aligned}
& \operatorname{Re}\left[H_{j P}(\omega)\right]\left(\left|\lambda_{k}\right|^{2}-\omega^{2}\right)+2 \omega \operatorname{Im}\left[H_{j P}(\omega)\right] \operatorname{Re}\left(\lambda_{k}\right) \\
& \quad=-2\left[\operatorname{Re}\left(\lambda_{k}\right) \operatorname{Re}\left(A_{j P, k}\right)+\operatorname{Im}\left(\lambda_{k}\right) \operatorname{Im}\left(A_{j P, k}\right)\right] \\
& \operatorname{Im}\left[H_{j P}(\omega)\right]\left(\left|\lambda_{k}\right|^{2}-\omega^{2}\right)-2 \omega \operatorname{Re}\left[H_{j P}(\omega)\right] \operatorname{Re}\left(\lambda_{k}\right) \\
& \quad=2 \omega \operatorname{Re}\left(A_{j P, k}\right) .
\end{aligned}
$$

Both equations are linear in four variables: $\left|\lambda_{k}\right|^{2}$, $\operatorname{Re}\left(\lambda_{k}\right), \operatorname{Re}\left(A_{j P, k}\right)$, and $\left[\operatorname{Re}\left(\lambda_{k}\right) \operatorname{Re}\left(A_{j P, k}\right)+\operatorname{Im}\left(\lambda_{k}\right) \operatorname{Im}\left(A_{j k}\right)\right] . \operatorname{In}$ principle, evaluating this pair of real equations at two arbitrary frequencies would yield four linear simultaneous equations that could be solved for the unknowns, from which the values of $\lambda_{k}$ and $A_{j P, k}$ could be extracted. In practice, the evaluation of Eqs. (12) and (13) at two frequencies is not sufficient for a variety of reasons. Hence, the strategy is to evaluate the equations at a multitude of frequencies, from which the four combination variables are determined by a linear least-squares procedure.

Not all of the FRF data is used to obtain the least squares solution. Selecting values increasingly far from the vicinity of a resonance enhances the contribution of other modes to the FRF, thereby defeating the notion that a single mode can be fit. Also, for additive white noise the best signal-to-noise ratios are at the resonance peaks. However, there are not likely to be many FRF values at a resonance unless the frequency increment is very fine. Furthermore, raising the number of values to be matched by using FRF values away from a resonance affords a greater opportunity for distributing the error. This dilemma was explored by Ginsberg et al. ${ }^{11}$ through a Monte Carlo study. They found that using all FRF points above the quarter-power points gave the best results for uniformly distributed random noise at several levels. This criterion is met by selecting for the least-squares fit only those values of $H_{j P}(\omega)$ that satisfy $\left|H_{j P}(\omega)\right| \geqslant \delta \max \left(\left|H_{j P}(\omega)\right|\right), \quad$ where $\delta=0.5$ yields the quarter-power points.

\section{IMPROVEMENT FOR LOW MODAL MOBILITY}

In the original conception of AMI the residue factors $A_{j P, k}$ obtained from a set of FRF data were used to calculate normal modes according to

$$
U_{P k}=\left(\frac{A_{P P, k}}{\lambda_{k}}\right)^{1 / 2}, \quad U_{j k}=\left(\frac{A_{j P, k}}{\lambda_{k} U_{P k}}\right), \quad j \neq P .
$$

A problematic aspect of the preceding arises if a generalized displacement has a near nodal value in some mode. Let $J$ denote the number of this generalized displacement and let $K$ denote the mode at which this condition occurs. Because
$U_{J K}$ is very small, the signal-to-noise ratio for $H_{J P}(\omega)$ will be poor when $\omega \approx \Omega_{K}$.

Consequently, there will be no evidence of the $K$ th mode's contribution when the FRF dataset for displacement $J$ is processed, resulting in a missing value of $A_{J P, k}$ and failure to estimate $\lambda_{K}$ from that FRF. Two possibilities exist. The simpler situation corresponds to $J \neq P$, that is, the nearnodal measurement point is not the location where the system was driven. If the transfer functions for other displacements in the vicinity of $\omega=\Omega_{K}$ have reasonable signal-tonoise ratios, then processing the $H_{j P}(\omega)$ datasets for $j \neq J$ will lead to a reasonable estimate for $\lambda_{K}$ and $A_{j P, K}$. As explained previously, averaging the estimates of $\lambda_{K}$ will ignore the missing value. Also, the missing residue factor $A_{J P, K}$ may be considered to be zero, so the normal mode coefficients may be computed according to Eqs. (14).

The situation is much worse if the near-nodal measurement point is the location where the system was excited, $J$ $=P$. If one were so unfortunate as to drive the system at a true modal node, so that $U_{P K}=0$, the $K$ th mode would not be excited. In that case, identifying mode $K$ would require redoing the measurements with a different drive point. The more likely circumstance is that $U_{P K}$ is very small, but nonzero. In that case smallness of the first denominator in Eq. (6) when $\omega_{m} \approx \Omega_{K}$ might lead to $H_{j P}(\omega)$ values for $j \neq P$ that have good signal-to-noise ratios in the vicinity of $\omega_{K}$. This would make it possible for AMI to estimate $\lambda_{K}$ and $A_{j P, K}$ for $j \neq P$ from those other displacements. However, the failure to identify mode $K$ from the data for displacement $P$ would leave the coefficient $A_{P P, K}$ undetermined. It therefore would not be possible to identify the normalized mode coefficients according to Eqs. (14).

In the last case, one could readily extract an estimate of a relative mode shape according to

$$
U_{n K} / U_{j K} \equiv A_{n P, K} / A_{j P, K} .
$$

Here $A_{j P, K}$ is a residue factor that has been identified as being nonzero, and $A_{n P, k}$ would be considered to be zero for any missing values. However, a simple change in the strategy for using AMI ensures that if an estimate of $\lambda_{K}$ is obtained from any FRF dataset, then an estimate for all corresponding normal mode coefficients will be obtained. Some existing algorithms in their later stages use known eigenvalues to estimate eigenvectors through a global fit of all FRF data; see Richardson and Formenti, ${ }^{9}$ for example. This notion is eminently suitable for the present situation. If the eigenvalues are taken to be known, then the only unknowns in Eq. (6) are the $A_{j P, k}$ coefficients, which occur there linearly. Many values of $H_{j P}(\omega)$ are available, corresponding to numerous frequencies and each of the displacements. Therefore, the right side of Eq. (6) can be matched to the measured values of $H_{j P}(\omega)$ in a linear least-squares procedure, which is relatively efficient, and not iterative. The result is that a value is obtained for every $A_{j P, k}$ coefficient for which a value of $\lambda_{k}$ has been estimated, and consequently that an estimate for each mode vector will be obtained.

To implement the procedure in the current context, each FRF is broken into real and imaginary parts, which leads to an error function for each part. The squared errors to be 
minimized are the inner products of the total error vector formed from the individual errors at each frequency,

$$
\begin{aligned}
R_{j}^{(1)}= & \sum_{m=1}^{M}\left[\operatorname{Re}\left(H_{j P}\left(\omega_{m}\right)\right)\right. \\
& \left.-\operatorname{Re} \sum_{k=1}^{N}\left(\frac{A_{j P, k}}{i \omega_{m}-\lambda_{n}}+\frac{A_{j P, k}^{*}}{i \omega_{m}-\lambda_{k}^{*}}\right)\right]^{2}, \\
R_{j}^{(2)}= & \sum_{m=1}^{M}\left[\operatorname{Im}\left(H_{j P}\left(\omega_{m}\right)\right)\right. \\
& \left.-\operatorname{Im} \sum_{k=1}^{N}\left(\frac{A_{j P, k}}{i \omega_{m}-\lambda_{k}}+\frac{A_{j P, k}^{*}}{i \omega_{m}-\lambda_{k}^{*}}\right)\right]^{2} .
\end{aligned}
$$

As was done for the SDOF identification of the eigenvalues, the frequencies $\omega_{m}$ are selected to be close to each identified natural frequency, so that the $H_{j P}\left(\omega_{m}\right)$ used for the fit have the best signal-to-noise ratio. The presence of the residue factors and their complex conjugates in the preceding is most readily handled by considering the real and imaginary parts of each $A_{j P, k}$ to be distinct unknowns. Applying the linear least squares procedure to $R_{j}^{(1)}$ and $R_{j}^{(2)}$ individually for a specific $j$ yields both parts of $A_{j P, k}$ for $k=1, \ldots, N$. Hence, a full evaluation of the normal modes entails repeating the procedure for each FRF dataset, $j=1, \ldots, N_{0}$. The normal mode coefficients are then calculated according to Eqs. (14).

\section{DISCUSSION}

The algorithm of mode isolation (AMI) is an iterative procedure for extracting modal properties from frequency response functions (FRFs). Each FRF is fit to the analytical form, which consist of a sequence of poles that define the natural frequencies and modal damping ratios, and residue factors, from which the mode vectors are extracted. Each step of the two iterative phases focuses on a single mode's contribution.

The latest version of AMI uses linear least-squares routines to identify that mode's eigenvalue and residue factors. Separate identifications are performed on each FRF, leading to multiple estimates. The eigenvalues obtained from each FRF are averaged using a specified merging criterion. Instead of using the residue factors obtained by processing the individual FRFs, the third phase of the procedure uses the average eigenvalues as inputs to a linear least-squares identification that yields a global estimate of all residue factors. These modifications improve the accuracy, as well as the computational efficiency of the identification, because linear least-squares is a not iterative, and it does not depend on having a good initial guess for the desired parameters.

The computational efficiency of AMI also is enhanced by the fact that only a subset of the FRF data is processed. The frequency increment for a FRF must be a fraction of the smallest modal bandwidth, in order to recognize the existence of all modes. Consequently, the number of frequency samples is inherently very large in comparison to the number of modes to be identified. MDOF identification algorithms in current use estimate the parameters by fitting all of the re- sponse data to the modal representation. As a result, the associated computational effort is scaled by the size of the dataset. In contrast, the effort entailed in AMI's identification processes is scaled by the relatively small number of FRF values that reside close to resonance peaks. In fact, the bulk of operations for AMI are devoted to forming the subtraction and isolation residuals.

Another aspect of the question of efficiency revolves around the need to identify as part of the overall process the number of modes whose natural frequency resides in the frequency interval of interest. Other MDOF techniques begin with an a priori guess for the number of modes, so that multiple such guesses are usually tried in order to identify which modes are computational artifacts. In AMI, the number of modes is obtained directly as part of the overall procedure. As a result, the FRF data is processed only once.

One overall limitation of AMI that was identified concerns modes that are very closely spaced. Trials using data for a two-degree-of-freedom system whose FRF are noisefree indicated that the two complex modes could not be identified if their natural frequencies differed by less than $40 \%$ of the average of their bandwidth. Recognition of this limitation was built into the general procedure. When AMI is applied to systems whose modes have this characteristic, the outcome is the identification of a single mode whose properties match the combined FRF contributions of the individual modes. In general, the robust identification of modes whose natural frequencies are extremely close requires a MIMO procedure. Work on such a version of AMI is presently underway.

\section{ACKNOWLEDGMENT}

This material is based on work supported under a National Science Foundation Graduate Research Fellowship.

${ }^{1}$ N. M. M. Maia, J. M. M. Silva, J. He, N. A. J. Lieven, R. M. Ling, G. W. Skingle, W.-T. To, and A. P. V. Urgueira, Theoretical and Experimental Modal Analysis and Testing (Research Studies Press, Ltd., Taunton, Somerset, England, 1997), Chap. 4.

${ }^{2}$ D. J. Ewins, Modal Testing: Theory, Practice and Applications, 2nd edition (Research Studies Press Ltd., Baldock, Hertfordshire, England, 2001), Chap. 4.

${ }^{3}$ R. J. Allemeng and D. L. Brown, "A unified matrix polynomial approach to modal identification," J. Sound Vib. 211, 301-322 (1998).

${ }^{4}$ S. W. Doebling, K. F. Alvin, and L. D. Peterson, "Limitations of statespace system identification algorithms for structures with high modal density," Proceedings of the 12th International Modal Analysis Conference, Honolulu, HI, 1994, pp. 633-637.

${ }^{5}$ M. V. Drexel and J. H. Ginsberg, "Mode isolation: a new algorithm for modal parameter identification," J. Acoust. Soc. Am. 110, 1371-1378 (2001).

${ }^{6}$ Y.-D. Joh and C.-W. Lee, "Excitation methods and modal parameter identification in complex modal testing of rotating machinery," Int. J. Anal. Exp. Modal Anal. 8, 179-203 (1993).

${ }^{7}$ M. V. Drexel and J. H. Ginsberg, "Modal parameter identification using state space mode isolation," Proceedings of the 19th International Modal Analysis Conference, Orlando, FL, 2001.

${ }^{8}$ M. V. Drexel, J. H. Ginsberg, and B. R. Zaki, "State space implementation of the algorithm of mode isolation," Trans. ASME, J. Vib. Acoust. 125, 205-213 (2003).

${ }^{9}$ M. H. Richardson and D. L. Formenti, "Global curve-fitting of frequency response measurements using the rational fraction polynomial method," Proceedings of the 3rd International Modal Analysis Conference, Orlando, FL, 1985.

${ }^{10}$ B. R. Zaki, A Modified Approach to Improve the Robustness of the Algo- 
rithm of Mode Isolation, Ph.D. thesis, Georgia Institute of Technology, Atlanta, GA, 2002

${ }^{11}$ J. H. Ginsberg, M. Allen, A. Ferri, and C. Moloney, "A general linear least squares sdof algorithm for identifying eigenvalues and residues," Pro- ceedings of the 21st International Modal Analysis Conference, Orlando, FL, 2003.

${ }^{12}$ J. H. Ginsberg, Mechanical and Structural Vibrations: Theory and Applications (Wiley, New York, 2001), Chap. 10. 